\title{
A case of mucocutaneous leishmaniasis
}

\author{
Pietro Galioto, MD \\ Vincenzo Fornaro, MD
}

\begin{abstract}
Leishmaniasis is prevalent in most warm-climate areas of the world. We describe a long-undiagnosed case of mucocutaneous leishmaniasis in Italy that might have been contracted in Costa Rica. The patient's signs and symptoms included granulomatous-like lesions on the forehead and legs, nasal obstruction accompanied by serous and crusted rhinorrhea, and diffuse granulomatous-like lesions in the septum and turbinates. The patient was treated with three on-off cycles of itraconazole. At the 1-year follow-up, all his lesions had nearly disappeared.
\end{abstract}

\section{Introduction}

Leishmaniasis is a parasitic disease that is endemic in Europe, the Middle East, India, central Asia, central and northern Africa, Central America, and South America. ${ }^{1}$ In Italy, it is mainly found in the central and southern regions and in Sicily and Sardinia.

The visceral form is found in all the areas of the world mentioned above. ${ }^{2}$ The cutaneous form, called Old World leishmaniasis, is widespread in the Middle East, in most Mediterranean countries, and in India. ${ }^{3}$ The mucocutaneous form, called New World leishmaniasis, is limited to Central and South America. ${ }^{4}$ In this article, we describe a case of mucocutaneous leishmaniasis that appeared in Italy and that was probably contracted in Costa Rica.

\section{Case report}

In January 1998, we examined a 60-year-old man who had come to us for an evaluation of painless lesions on his forehead and legs. He noted that he had visited Costa Rica in early 1996 and that he developed an intermittent fever similar to that of influenza ( 38 to $38.5^{\circ} \mathrm{C}$ ) 3 weeks after he had returned to Italy. He also began to experience a loss of appetite, chronic fatigue, and intermittent diarrhea. The lesions first appeared approximately 1 year later, in January 1997. In January 1998, he developed a bilateral nasal obstruction that was accompanied by se-

From the Department of Otorhinolaryngology, University of Pavia School of Medicine, Pavia, Italy.

Reprint requests: Pietro Galioto, MD, via S. Giovanni in Borgo No. 11, 27100 Pavia, Italy. Phone: +39-335-602-5321; fax: +39-038224627; e-mail: piegalio@tin.it rous and crusted rhinorrhea and came to see us.

On physical examination, we noted that granulomatous-like lesions were present on the forehead and on the anterior region of the tibia on both legs (figure 1). Further examination and testing revealed that the patient had hepatosplenomegaly and several abnormal laboratory test results, including an elevated erythrocyte sedimentation rate, a certain degree of anemia, and a slight alteration in kidney function.

Nasal endoscopy revealed a hyperemic mucosa and diffuse granulomatous-like lesions in the septum and turbinates (figure 2). The remainder of the cavity was substantially undamaged. With the aid of videoendoscopy, a biopsy specimen of the mucosal lesions was obtained for culture and microscopic examination. Leishmania parasites were isolated on culture, and they were identified as Leishmania panamensis (figure 3). Microscopic examination yielded negative results. Histopathology identified inflammatory infiltrates and some microorganisms whose characteristics were suggestive of Leishmania.

Noncontrast axial and coronal computed tomography (CT) of the paranasal sinuses demonstrated rhinosinus involvement, with opacification of the maxillary and ethmoid sinuses and an inflammatory reaction in the mucosa (figure 4).

The patient was treated with itraconazole for 8 weeks, then the drug was stopped for 3 months. This cycle was repeated twice more.

One year later, physical examination revealed that the patient experienced a marked improvement and the skin lesions had almost disappeared. Follow-up nasal endoscopy showed that the crusted lesions had also nearly disappeared, and this had improved the nasal airflow. Moreover, the patient reported an improvement in his general overall health.

\section{Discussion}

We know from the literature that domestic dogs, some rodents, and Candidae are essentially the natural hosts for Leishmania organisms and as such are the source of infection. ${ }^{5}$ The only exception to this occurs in India, where most cases are traced to a human host. ${ }^{6}$

Infection is indirect in that it is transmitted by numerous species of insects of the genera Phlebotomus and 
Lutzomyia. The female insect becomes infected when it bites an infected mammal, and then the insect passes on the infection when it bites a human.

The characteristics of leishmaniasis include a propensity toward males, the presence of large or multiple primary lesions, and a persistent duration of the lesions ( $>1 \mathrm{yr}$ ). In most cases, hematogenous or lymphatic dissemination is responsible for the diffusion of mucocutaneous leishmaniasis to other mucosal districts. On occasion, mucous membrane involvement develops as a result of the direct extension of nearby skin lesions. ${ }^{7}$ The mucosal lesions of the nasal cavity involve the turbinates, principally the middle turbinates. The nasal septum then becomes infiltrated and inflamed, and it eventually perforates.

The mucocutaneous form of leishmaniasis has a certain predilection to the distal cartilaginous part of the nose. Once established there, it can cause a deformity known as tapir's nose or camel's nose. ${ }^{8}$ The destruction can spread to the palate, pharynx, tonsils, and lips. Bony structures remain intact. Invasion of the respiratory tract can affect the larynx, trachea, and bronchi.

Diagnosis. It is difficult to diagnose leishmaniasis at an early stage for two reasons. First, it is not a common

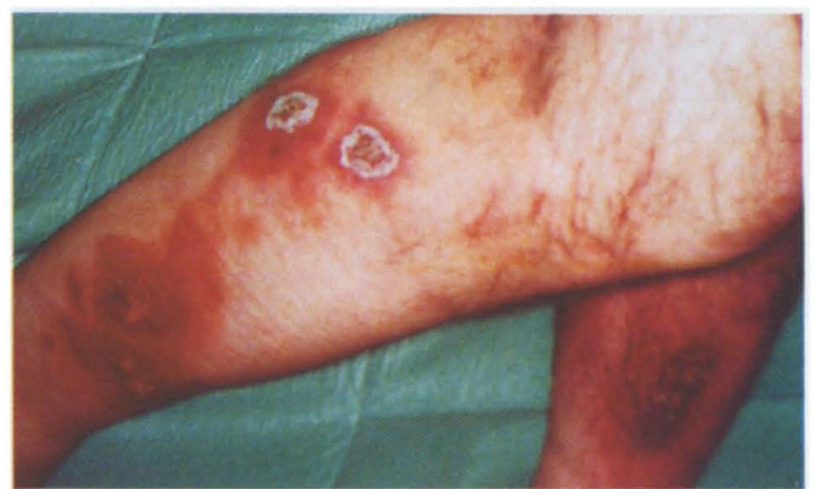

Figure 1. Physical examination reveals the presence of granulomatous-like lesions on the anterior region of the tibia on both legs.

disease. Second, the lesions that develop in the cutaneous and mucocutaneous forms are nonspecific erythematous papules that in the final stage of their development resemble granulomatous lesions. Therefore, these lesions can be confused with those found in Wegener's granulomatosis (which is also marked by cutaneous manifestations and can involve the nasal mucesa), leprosy, and tuberculosis. ${ }^{9}$

\section{Reach More Patients.}

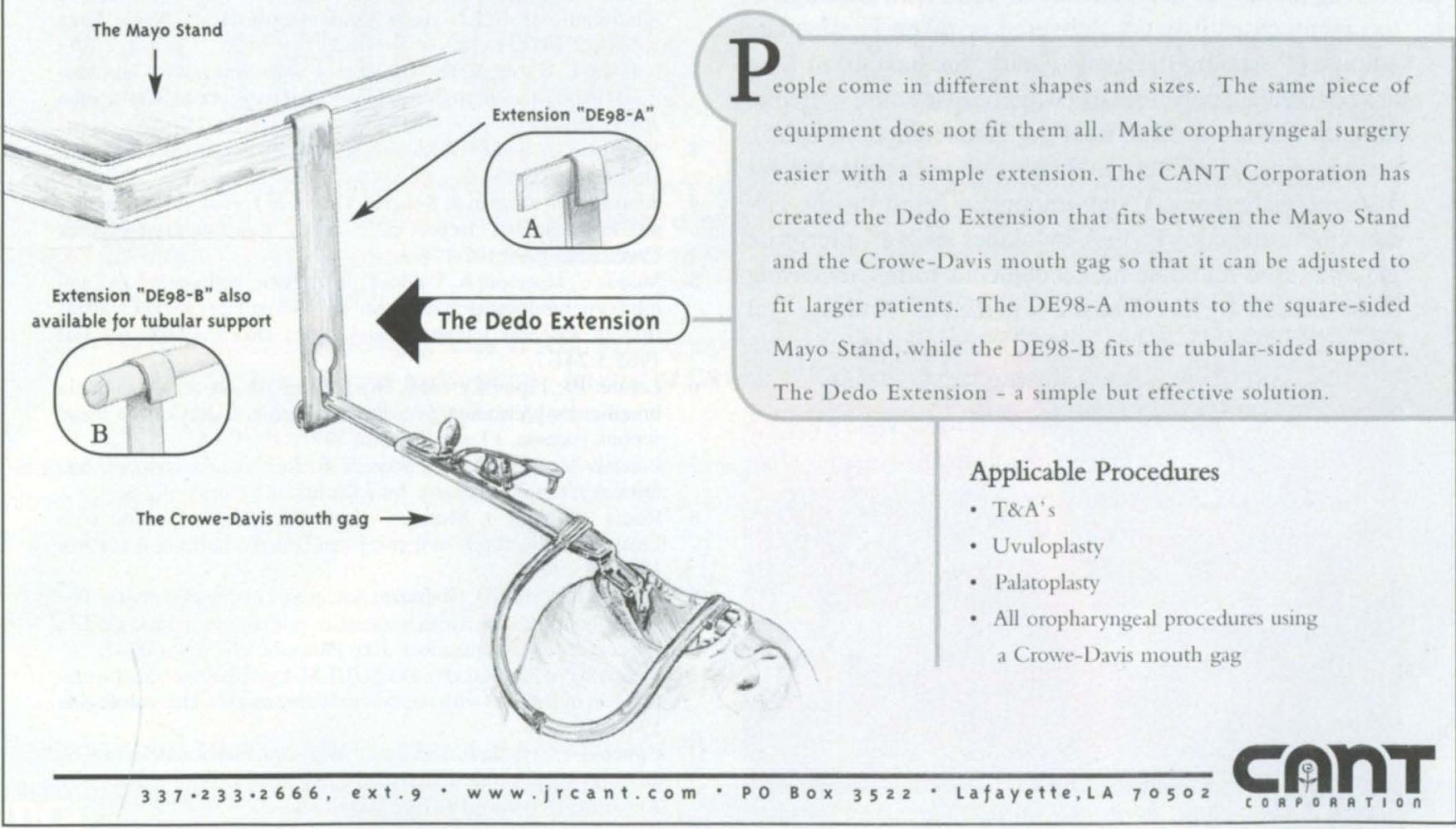

For more information Circle 115 on Reader Service Card 


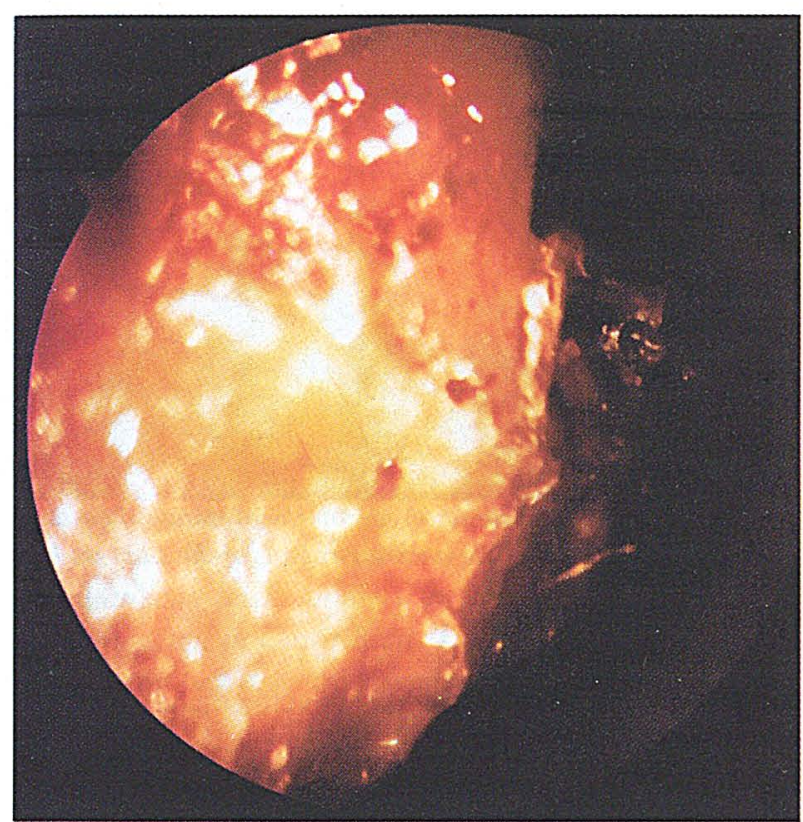

Figure 2. Nasal endoscopy detects granulomatous-like crusted lesions in the nasal septum.

Cultures and recombinant immunoenzymatic testing can detect the presence of Leishmania amastigotes and facilitate the differential diagnosis. ${ }^{10}$

Treatment. Left untreated, mucocutaneous leishmaniasis leads to malnutrition and acute respiratory insufficiency, which are the two primary causes of death in fatal cases.

Drug therapy is the mainstay of treatment, although in too many cases it is not delivered or taken in adequate amounts. ${ }^{11}$ Among the many agents that have been used are pentavalent antimonials (which cause some systemic toxicity and are used to treat the mucocutaneous form), pentamidine, amphotericin B (which is used primarily for visceral leishmaniasis), paromomycin (used for the visceral and cutaneous forms), cytokines such as interferon gamma (used for some mucocutaneous forms, including those caused by Leishmania braziliensis strains), oral

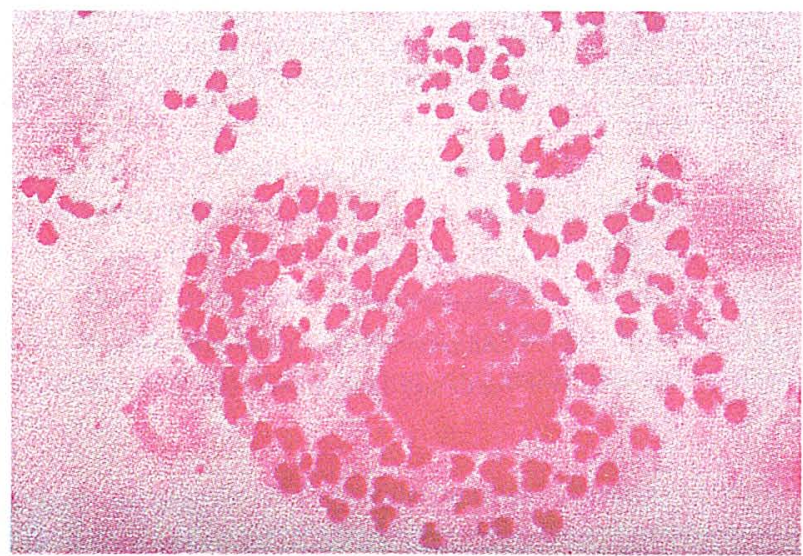

Figure 3. On culture, intra- and extracellular organisms are identified as Leishmania panamensis.

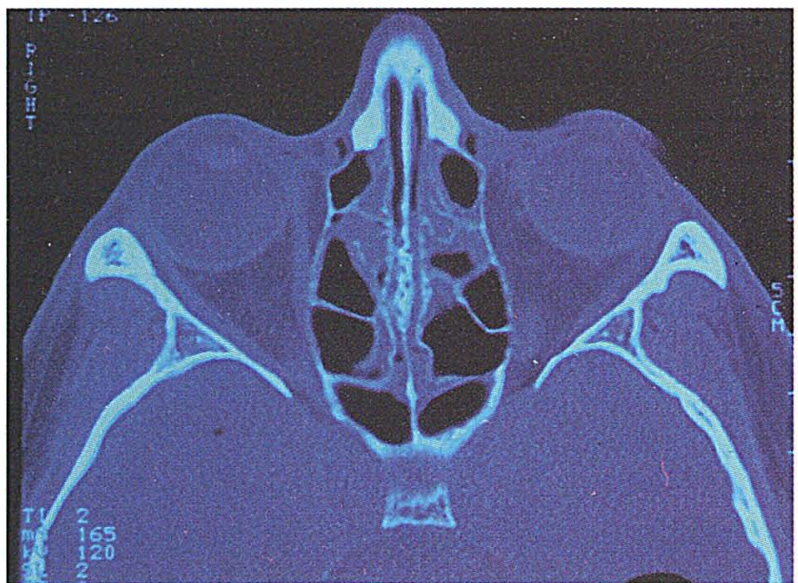

Figure 4. Noncontrast axial CT shows the partial opacification of the ethmoid.

antifungals such as ketoconazole, fluconazole, and itraconazole (which are well tolerated), and hipoxanthine (which inhibits purine anabolism and slows Leishmania proliferation). ${ }^{12}$

It is our hope that otolaryngologists will keep leishmaniasis in mind when examining lesions of the nasal mucosa, especially as the world continues toward globalization of its peoples. This is particularly important when we examine patients with nasal obstruction and serous and crusted rhinorrhea who have a travel history to other countries, especially South America and the Middle East.

\section{References}

1. Grunwald MH, Amichai B. Pathologic quiz case 1. Cutaneous leishmaniasis (CL). Arch Otolaryngol Head Neck Surg 1996;122:1412, 1414.

2. Patuano E, Carrat X, Drouet $Y$, et al. [Mucocutaneous leishmaniasis in otorhinolaryngology]. Ann Otolaryngol Chir Cervicofac 1993;110:415-9.

3. Vernham GA, SadiqH, MallonEA. Nasal leishmaniasis. JLaryngol Otol 1993;107:834-6.

4. Khayat CM, Perreau P, Souchal-Delacour I, et al. [Mucocutaneous leishmaniasis. Report of 2 cases]. Ann Otolaryngol Chir Cervicofac 1990;107:494-9.

5. Medda C, Maccioni A, Valdes E. [Cytologic, histopathologic and ultrastructural research on a case of nasal and laryngeal localization of visceral leishmaniasis]. Acta Otorhinolaryngol Ital 1985;5:241-7.

6. Lohuis PJ, Lipovsky MM, Hoepelman AL, et al. Leishmania braziliensis presenting as a granulomatous lesion of the nasal septum mucosa. J Laryngol Otol 1997;111:973-5.

7. Landau M, Srebrnik A, Brenner S. Leishmaniasis recidivans mimicking lupus vulgaris. Int J Dermatol 1996;35:572-3.

8. Roura i Moreno J, Moragas i Lluis M. [Nasal infection with Leishmania. Apropos of a case]. An Otorrinolaringol Ibero Am 1988;15:291-8.

9. Githure JI, Reid GD, Binhazim AA, et al. Leishmania major: The suitability of East African nonhuman primates as animal models for cutaneous leishmaniasis. Exp Parasitol 1987;64:438-47.

10. Pagnano PM, da Costa JC, Bechelli LM. Lymphocyte transformation test in patients with American leishmaniasis. Dermatologica 1985;170:22-6.

11. Cohen HA, Livshin R. Treatment of leishmaniasis nodosa (oriental sore) with intralesionally injected emetine hydrochloride. J Am Acad Dermatol 1987;17:595-9.

12. Rosbotham JL, Corbett EL, Grant HR, et al. Imported mucocutaneous leishmaniasis. Clin Exp Dermatol 1996;21:288-90. 